\title{
How development affects evolution
}

2 Mauricio González-Forero ${ }^{1 *} \&$ Andy Gardner ${ }^{1}$

${ }^{3}{ }^{1}$ School of Biology, University of St Andrews, Dyers Brae, St Andrews KY16 9TH, UK

$4 \quad *$ e-mail: mgf3@st-andrews.ac.uk

5 Natural selection acts on developmentally constructed phenotypes, raising the question of how development affects evolution. A mathematical framework that explicitly integrates development into evolution has recently been derived. Here we use this framework to analyse how development affects evolution. We show that, whilst selection pushes genetic and phenotypic evolution uphill on the fitness landscape, development determines the admissible evolutionary pathway, such that evolutionary outcomes occur at path peaks, which need not be peaks of the fitness landscape. Development can generate path peaks, triggering adaptive radiations, even on constant, single-peak landscapes. Phenotypic plasticity, niche construction, extra-genetic inheritance, and developmental bias variously alter the evolutionary path and hence the outcome. Selective development, whereby phenotype construction may point in the adaptive direction, may induce evolution either towards or away landscape peaks depending on the developmental constraints. Additionally, developmental propagation of phenotypic effects over age allows for the evolution of negative senescence. These results help explain empirical observations including punctuated equilibria, the paradox of stasis, the rarity of stabilizing selection, and negative senescence, and show that development has a major role in evolution. 
been understood to affect evolution by shaping genetic covariation upon which selection acts ${ }^{1-5}$.

However, progress on understanding how development affects evolution has been difficult. A key reason is that equations describing phenotypic evolutionary change have until recently not been derived from an explicit account of development, but from considerations of genetic covariation defined in terms of linear regression of trait values on gene content ${ }^{1,6}$. As regression describes relationships regardless of their underlying mechanisms, this approach to genetic covariation may have limited a mechanistic understanding of the nature of genetic covariation arising from development.

There are longstanding calls for explicit integration of development into evolution ${ }^{7-13}$. These calls have recently highlighted several developmental factors_including phenotypic plasticity ${ }^{10}$, niche construction $^{14}$, extra-genetic inheritance ${ }^{7}$, and developmental bias ${ }^{15}$-as possibly having important, yet unrecognised, evolutionary consequences ${ }^{12,13}$. Calls for synthesis between developmental and evolutionary biology have also asked for consideration of the mechanistic basis of phenotype construction, non-linear genotype-phenotype maps, gene-gene and geneenvironment interactions, non-normal distributions, far-from-equilibrium evolutionary dynamics, dynamic fitness landscapes, evolution and the nature of the G-matrix, evolvability and epigenetics, and a variety of other complexities ${ }^{11,16}$. A new mathematical framework integrates explicit development into evolution while incorporating all of these elements by building upon many advances in evolutionary modelling over the last decades ${ }^{17}$. Here we use this framework to examine how development affects evolution. 


\section{Results}

Framework overview. In the framework, each individual is described at each age by her gene expression level for a set of gene products, by her trait values for a set of developed phenotypes, and by the values of a set of environmental variables describing the local environment that the individual faces. The gene expression level across all gene products of a mutant individual of age $a$ is described the vector $\mathbf{y}_{a}=\left(y_{1 a}, \ldots, y_{N_{\mathrm{c}} a}\right)^{\top}$ of her $N_{\mathrm{c}}$ gene products (control variables). The developed phenotype of a mutant individual of age $a$ is described by the vector $\mathbf{x}_{a}=\left(x_{1 a}, \ldots, x_{N_{\mathrm{s}} a}\right)^{\top}$ of her $N_{\mathrm{s}}$ phenotypes (state variables). The environment of a mutant individual of age $a$ is described by the vector $\boldsymbol{\epsilon}_{a}=\left(\epsilon_{1 a}, \ldots, \epsilon_{N_{\mathrm{e}} a}\right)^{\top}$ of her $N_{\mathrm{e}}$ mutually independent variables describing the individual's environment (e.g., ambient temperature, which the individual may adjust, such as by roosting in the shade). A mutant individual's phenotype, gene expression, and environment across all ages are described respectively by $\mathbf{x}=\left(\mathbf{x}_{1} ; \ldots ; \mathbf{x}_{N_{\mathrm{a}}}\right)$, $\mathbf{y}=\left(\mathbf{y}_{1} ; \ldots ; \mathbf{y}_{N_{\mathrm{a}}}\right)$, and $\boldsymbol{\epsilon}=\left(\mathbf{y}_{1} ; \ldots ; \mathbf{y}_{N_{\mathrm{e}}}\right)$ (the semicolon indicates a linebreak). For ease of reference, we aggregate genotype and phenotype into a single vector called the "geno-phenotype". Thus, the geno-phenotype of a mutant individual of age $a \in\left\{1, \ldots, N_{\mathrm{a}}\right\}$ is described by the blockcolumn vector $\mathbf{z}_{a}=\left(\mathbf{x}_{a} ; \mathbf{y}_{a}\right)$. The mutant geno-phenotype across all ages is $\mathbf{z}=(\mathbf{x} ; \mathbf{y})$. We similarly aggregate genotype, phenotype, and environment into a single vector called the "genoenvo-phenotype". The geno-envo-phenotype of a mutant individual of age $a \in\left\{1, \ldots, N_{\mathrm{a}}\right\}$ is described by the block-column vector $\mathbf{m}_{a}=\left(\mathbf{z}_{a} ; \boldsymbol{\epsilon}_{a}\right)$. The mutant geno-envo-phenotype across all ages is $\mathbf{m}=(\mathbf{z} ; \boldsymbol{\epsilon})$. Resident (i.e., wild-type) variables are denoted analogously with an overbar (e.g., $\overline{\mathbf{z}})$. 
To incorporate development, we describe an individual's phenotype at a given age as a function of all her traits at the immediately preceding age and of the social environment experienced at that age. Thus, the phenotype of a mutant individual at age $a+1$ satisfies the developmental constraint

$$
\mathbf{x}_{a+1}=\mathbf{g}_{a}\left(\mathbf{m}_{a}, \overline{\mathbf{z}}\right),
$$

where the developmental map $\mathbf{g}_{a}\left(\mathbf{m}_{a}, \overline{\mathbf{z}}\right)=\left(g_{1 a}\left(\mathbf{m}_{a}, \overline{\mathbf{z}}\right), \ldots, g_{N_{\mathrm{s}} a}\left(\mathbf{m}_{a}, \overline{\mathbf{z}}\right)\right)^{\top}$ depends on the mutant's gene expression, phenotype, and environment at age $a$ and on the gene expression and phenotype of social partners of any age. The developmental map describes the developmental constraints on the phenotype $\mathbf{x}$ which cannot take arbitrary values but only those satisfying Eq. (1). The developmental map can evolve and take any (differentiable) form as gene expression, phenotype, and environment evolve.

An individual's environment may depend on her own gene expression and phenotype, on the gene expression and phenotype of social partners, and on exogenous processes. The dependence of the environment on the gene expression and phenotype of herself and social partners can describe niche construction by her or her social partners. The dependence of the environment on exogenous processes can describe, for instance, eutrophication or climate change caused by members of other species. Thus, a mutant's environment at age $a$ satisfies the environmental constraint

$$
\boldsymbol{\epsilon}_{a}=\mathbf{h}_{a}\left(\mathbf{z}_{a}, \overline{\mathbf{z}}, \tau\right)
$$

where the environmental map $\mathbf{h}_{a}\left(\mathbf{z}_{a}, \overline{\mathbf{z}}, \tau\right)=\left(h_{1 a}\left(\mathbf{z}_{a}, \overline{\mathbf{z}}, \tau\right), \ldots, h_{N_{\mathrm{e}} a}\left(\mathbf{z}_{a}, \overline{\mathbf{z}}, \tau\right)\right)^{\top}$ depends on the mutant's gene expression and phenotype at that age (e.g., from behavioural choice or environmental modification), on her social partners' gene expression and phenotypes at any age (e.g., from 
social niche construction), and on the evolutionary time $\tau$ due to exogenous environmental change. Consequently, the environment $\boldsymbol{\epsilon}$ can only take values allowed by the environmental map $\mathbf{h}$ describing the environmental constraints. In turn, a mutant's fertility $f_{a}\left(\mathbf{m}_{a}, \overline{\mathbf{m}}\right)$ and survival probability $p_{a}\left(\mathbf{m}_{a}, \overline{\mathbf{m}}\right)$ at age $a$ depend on her gene expression, phenotype, and environment at that age, and on the gene expression, phenotype, and environment of her social partners of any age.

The developmental constraint (1) incorporates: developmental bias, in that the phenotype $\mathbf{x}$ may be predisposed to develop in certain ways; phenotypic plasticity, in that the same genotype $\mathbf{y}$ can generate a different phenotype $\mathbf{x}$ under a different environment $\boldsymbol{\epsilon}$; adaptive phenotypic plasticity, for example, via somatic selection or reinforcement learning (e.g., if $g_{i a}$ is proportional to the gradient $\partial g_{j a} / \partial x_{i a}$ of a material payoff $g_{j a}$ earned at age $a$ that increases fitness); niche construction, in that environmental variables depend on developmentally constructed traits of the individual and social partners; and extra-genetic inheritance, in that an individual's phenotype at age $a+1$ can be an identical or modified copy of non-relatives' phenotypes. Moreover, the developmental constraint (1) can evolve, be non-linear, and mechanistically describe development, gene-gene interaction, and gene-environment interaction. For illustration, we allow for the possibility that the developmental map $\mathbf{g}_{a}$ depends on the selection gradient $\partial w / \partial \mathbf{z}_{a}$ (defined below), in which case we say that development is selective.

Development blocks evolutionary change. We investigate how development as described by Eq. 1 affects evolution by using equations that yield a well-defined description of the long-term evolution of a developed phenotype $\overline{\mathbf{x}}$ as the climbing of a fitness landscape. Assuming for now 
that there is (i) no niche construction $\left(\partial \boldsymbol{\epsilon}^{\top} / \partial \mathbf{z}=\mathbf{0}\right)$, (ii) no social development $\left(\mathrm{d} \mathbf{x}^{\top} / \mathrm{d} \overline{\mathbf{z}}=\mathbf{0}\right)$, and (iii) no exogenous environmental change $(\partial \boldsymbol{\epsilon} / \partial \tau=\mathbf{0})$, then such well-defined description of long-term phenotypic evolution is given by

$$
\frac{\mathrm{d} \overline{\mathbf{z}}}{\mathrm{d} \tau}=\kappa \mathbf{G}_{\mathbf{z}} \frac{\partial w}{\partial \mathbf{z}}
$$

provided that the developmental constraint (1) is satisfied at all times (derivatives are evaluated at $\mathbf{y}=\overline{\mathbf{y}}$ throughout; $\boldsymbol{k}$ is a positive scalar proportional to the mutation rate and the carrying capacity). To yield a well-defined description of long-term phenotypic evolution as the climbing of a fitness landscape, Eq. (3) simultaneously describes genetic and phenotypic evolution since $\mathbf{z}=(\mathbf{x} ; \mathbf{y})$ is both gene expression and phenotype (in this framework, gene expression plays the role of allele frequency). Eq. (3) has the same form of the Lande equation ${ }^{1}$, which describes short-term phenotypic evolution (the Lande equation is essentially the same as Eq. (3) replac$\operatorname{ing} \mathbf{z}$ for $\mathbf{x}$, where $\mathbf{G}_{\mathbf{x}}$ is a function of genetic traits $\overline{\mathbf{y}}$; Supplementary Information section 1).

Although Eq. (3) has the same form of the Lande equation ${ }^{1}$, it has been derived from an explicit account of development so we have formulas to relate development (1) to breeding value and so to genetic covariation $\mathbf{G}_{\mathbf{z}}$ that guarantee that the developmental constraint (1) is satisfied at all times. Because of age-structure, a mutant's relative fitness ${ }^{18}$ is

$$
w=\sum_{a=1}^{N_{\mathrm{a}}} w_{a}=\frac{1}{T} \sum_{a=1}^{N_{\mathrm{a}}}\left(\phi_{a} f_{a}+\pi_{a} p_{a}\right),
$$

where $T$ is generation time, and $\phi_{a}$ and $\pi_{a}$ are the forces ${ }^{19}$ of selection on fertility and survival at age $a ; T, \phi_{a}$, and $\pi_{a}$ depend on resident but not mutant values. Eq. (3) thus depends on the selection gradient $\partial w / \partial \mathbf{z}$, which measures direct directional selection on the geno-phenotype: it is a vector that points in the direction of steepest increase in fitness in geno-phenotype space. 
Additionally, the additive-genetic covariance matrix of the geno-phenotype is (cf. refs. ${ }^{6,20-23}$ )

$$
\mathbf{G}_{\mathbf{z}}=\frac{\mathrm{d} \mathbf{z}}{\mathrm{d} \mathbf{y}^{\top}} \mathbf{G}_{\mathbf{y}} \frac{\mathrm{d} \mathbf{z}^{\top}}{\mathrm{d} \mathbf{y}},
$$

where $\mathbf{G}_{\mathbf{y}}=\operatorname{cov}[\mathbf{y}, \mathbf{y}]$ is the mutational covariance matrix (Supplementary Information section 2). Although in Eq. (3) there may be plasticity, it has no effect since by assumptions (i) and (iii) niche construction and exogenous environmental change are absent so the environment remains constant.

Eq. (3) implies that there are always absolute genetic constraints to adaptation. Indeed, to yield a well-defined description of long-term phenotypic evolution as the climbing of a fitness landscape, Eq. (3) simultaneously describes genetic and phenotypic evolution, so the climbing of the fitness landscape is in geno-phenotype space rather than phenotype space only as in the classic Lande equation. Since geno-phenotype space is necessarily larger than genetic space, then genetic covariation in geno-phenotype is absolutely constrained by the genetic space (i.e., $\mathbf{G}_{\mathbf{z}}$ is always singular because $\mathrm{d} \mathbf{z}^{\top} / \mathrm{d} \mathbf{y}$ has fewer rows than columns). Specifically, there cannot be genetic variation in as many directions in geno-phenotype space as there are phenotypes. Along such directions evolutionary change is blocked. In other words, as developed traits have an indirect genetic basis, the ability of natural selection to shape them is constrained by the ability of genes to shape developed traits. In this sense, development can be seen as blocking evolutionary change in some directions. Therefore, evolutionary stasis $(\mathrm{d} \overline{\mathbf{z}} / \mathrm{d} \tau=\mathbf{0})$ can occur away from landscape peaks, with persistent direct directional selection $(\partial w / \partial \mathbf{z} \neq \mathbf{0}$; see also refs. ${ }^{24-26}$ ). The singularity of $\mathbf{G}_{\mathbf{z}}$ means that the evolutionary dynamics are strongly nonstandard; in particular, consideration of direct directional selection alone is not sufficient for predicting possible evolutionary outcomes. This singularity is also consistent with common 
empirical observations of evolutionary stasis in wild populations despite directional selection and genetic variation, termed the "paradox of stasis" $27-29$.

Development determines the path. Development can more specifically be seen as determining the admissible evolutionary path. Assume again that there is (i) no niche construction $\left(\partial \boldsymbol{\epsilon}^{\top} / \partial \mathbf{z}=\mathbf{0}\right)$, (ii) no social development ( $\mathrm{d} \mathbf{x}^{\top} / \mathrm{d} \overline{\mathbf{z}}=\mathbf{0}$ ), and (iii) no exogenous environmental change $(\partial \boldsymbol{\epsilon} / \partial \tau=\mathbf{0})$. Then, the evolutionary dynamics of the phenotype and gene expression are equivalently given by

$$
\frac{\mathrm{d} \overline{\mathbf{z}}}{\mathrm{d} \tau}=\kappa \mathbf{G}_{\mathbf{z y}} \frac{\mathrm{d} w}{\mathrm{~d} \mathbf{y}},
$$

again provided that the developmental constraint (1) is satisfied at all times. This equation now depends on the total selection gradient of gene expression (see also refs. ${ }^{30,31}$ )

$$
\frac{\mathrm{d} w}{\mathrm{~d} \mathbf{y}}=\frac{\mathrm{d} \mathbf{z}^{\top}}{\mathrm{d} \mathbf{y}} \frac{\partial w}{\partial \mathbf{z}}=\frac{\partial w}{\partial \mathbf{y}}+\frac{\mathrm{d} \mathbf{x}^{\top}}{\mathrm{d} \mathbf{y}} \frac{\partial w}{\partial \mathbf{x}},
$$

which measures total genetic selection, that is, total directional selection on genes considering the ability of genes to affect the phenotype (in contrast, $\partial w / \partial \mathbf{y}$ measures direct directional selection on genes without considering such ability). Moreover, the additive genetic crosscovariance matrix between the geno-phenotype and gene expression is

$$
\mathbf{G}_{\mathbf{z y}}=\frac{\mathrm{d} \mathbf{z}}{\mathrm{d} \mathbf{y}^{\top}} \mathbf{G}_{\mathbf{y}} .
$$

Crucially, the matrix $\mathrm{dz} / \mathrm{d} \mathbf{y}^{\top}$, which maps change in gene expression to change in phenotype, is non-singular. Thus, Eq. (6) implies that evolutionary stasis occurs when total genetic selection vanishes ( $\mathbf{d} w / \mathbf{d} \mathbf{y}=\mathbf{0})$ if there are no absolute mutational constraints (i.e., if $\mathbf{G}_{\mathbf{y}}$ is non-singular). That is, total genetic selection can identify evolutionary equilibria, even though direct directional selection typically cannot. From Eq. (7), we find that total genetic selection depends on 
the matrix $d \mathbf{x}^{\top} / d \mathbf{y}$, which corresponds to Fisher's additive effects of allelic substitution ${ }^{6}$ and to Wagner's developmental matrix ${ }^{21}$ (Extended Data Fig. 1). This means that evolutionary equilibria depend on development. Moreover, since the developmental constraints (1) must be satisfied at all times, then developmental constraints determine both the evolutionary equilibria and the admissible evolutionary trajectory.

To gain intuition, consider the following simple example. Let there be one phenotype $x$ and one gene product with expression level $y$, no social interactions, no niche construction, and no exogenous environmental change. Let age structure be collapsed such that development occurs instantaneously, and let there be no density dependence. By removing social interactions and density dependence, evolutionary change can be not only abstractly but also visually described as the climbing of a fitness landscape. Selection can be seen as "pushing" the geno-phenotype uphill on the fitness landscape of the geno-phenotype in the direction of steepest ascent, whereas genetic covariation diverts evolutionary change in a possibly less steep direction. If genetic constraints were not absolute (i.e., if $\mathbf{G}_{\mathbf{z}}$ were non-singular), the population would eventually reach a landscape peak, which is commonly implicitly or explicitly assumed. Yet, as the phenotype arises from the developmental constraint (1), genetic constraints are necessarily absolute (i.e., $\mathbf{G}_{\mathbf{z}}$ is singular), so adaptive evolution is restricted to an admissible path on the landscape where the developmental constraint is met (Fig. 1a-c; the computer code used to generate all the figures is in the Supplementary Information). Adaptive evolution may thus be understood as the climbing of the fitness landscape along an admissible path determined by development. The evolutionary process eventually reaches a path peak if there are no absolute mutational constraints (Fig. 1a-c). Selection response vanishes at path peaks which are not nec- 
essarily landscape peaks (Fig. 1a-c). The admissible path yields an elevation profile of fitness, namely, the total fitness landscape of gene expression, on which adaptation is constrained to occur, and can have peaks and valleys created by the developmental map (Fig. 1d-f). Hence, selection pushes gene expression uphill on the total fitness landscape of gene expression, but in this profiled landscape evolutionary change is not blocked provided that there are no absolute mutational constraints. Consequently, without absolute mutational constraints, evolutionary stasis generally occurs at a peak on the total fitness landscape of gene expression, even though this does not generally correspond to a peak on the fitness landscape of the phenotype and gene expression. The $\mathbf{G}_{\mathbf{z}}$-matrix evolves as an emergent property as the resident phenotype and gene expression evolve, and genetic variances and covariances may substantially increase or decrease if development is non-linear, rather than being approximately constant as is often considered under short-term evolution with an infinite number of loci ${ }^{6,32}$ which we do not assume (Fig. 1g-i). Ultimately, development constrains the path of adaptation and thus shapes its outcome (Fig. 1j-1). Overall, development has a dual role in adaptation by determining both evolutionary equilibria (blue lines in Fig. $1 \mathrm{j}-\mathrm{l}$ ) and the admissible evolutionary path (red line in Fig. $1 \mathrm{j}-1)$.

That evolution stops at path peaks rather than landscape peaks may help explain abundant empirical data. Empirical estimation of selection has found that direct stabilizing selection is rare relative to direct directional selection across thousands of estimates in more than 50 species $^{29,33}$. The rarity of stabilizing selection has been puzzling under the common assumption that evolutionary outcomes occur at landscape peaks, where stabilizing selection is prevalent ${ }^{2,34}$. In contrast, the rarity of stabilizing selection is consistent with evolutionary 
outcomes occurring at path peaks. Indeed, if path peaks occur outside landscape peaks, evolutionary outcomes occur with persistent direct directional selection (see also refs. ${ }^{24-26}$ ) and relatively weak direct stabilizing selection (because direct stabilizing selection is relatively strong only near landscape peaks). Thus, evolution stopping at path peaks outside landscape peaks helps explain the otherwise puzzling observation of rare stabilizing selection (see also ref. ${ }^{31}$ ).

Development can drive diversification. The evolution of phenotypic diversity is typically explained in terms of selection or drift ${ }^{35}$, but the relevance of development has been less clear. We find that development can drive the evolution of phenotypic diversity, even in a constant, single-peak fitness landscape, where selection provides an evolutionary force while development translates it into diversification. This can happen in two ways. First, if the developmental map changes inducing a shifted path peak, the population can evolve different phenotypes and gene expression (Extended Data Fig. 2a-f). Second, the developmental map may change, generating new path peaks, if it is or becomes non-linear, such that the population may evolve different phenotypes and gene expression depending on the initial conditions (Extended Data Fig. 2g-l). Mathematically, multiple path peaks can arise from non-linear development because it can generate bifurcations altering the number of admissible evolutionary equilibria even though the number of evolutionary equilibria remains the same (i.e., infinite). This is a particular but previously unrecognised form of evolutionary branching ${ }^{36}$, which may be called evo-devo branching, where the path creates a valley in the total fitness landscape of gene expression so there is disruptive total genetic selection ${ }^{31}$ (Fig. 1e). There may not be a valley in the fitness landscape so no disruptive direct selection on the phenotype or gene expression (Fig. 1b), hence evo-devo branching only requires first-order selection (first derivatives of in- 
vasion fitness) rather than the second-order selection (second derivatives) needed in classic evolutionary branching. Thus, evo-devo branching might occur more readily than classic evolutionary branching. Development can then lead to phenotypic diversification, even with a constant single-peak fitness landscape of the geno-phenotype. Consequently, adaptive radiations might arise from evolution of the developmental map.

Our results partly substantiate a classic explanation of punctuated equilibria in terms of developmental constraints, an explanation that has been dismissed in the light of previous theory. Punctuated equilibria refer to long periods of morphological stasis alternated by periods of rapid change as observed in the fossil record ${ }^{37,38}$. The dominant explanation for punctuated equilibria is that fitness landscapes remain constant for long periods so there is stabilizing selection at an unconstrained optimum, and fitness landscapes subsequently undergo change $\mathrm{e}^{2,34}$. A classic alternative explanation is that (a) developmental constraints prevent change for long periods and (b) revolutions in the developmental program bring sudden change ${ }^{39}$. The ability of constraints to prevent change has been thought to be refuted by the common observation of selection response, sometimes under seeming developmental constraints ${ }^{40}$, while revolutions in the developmental program have been refuted on other grounds ${ }^{2,5,41}$. Our results substantiate the former, showing that selection response is consistent with developmental constraints preventing change: indeed, even if developmental constraints halt a population at a path peak, selection response is possible since path peaks may change, either by change in the path (e.g., by plasticity) or the landscape (e.g., by artificial selection). Thus, available evidence does not necessarily rule out that developmental constraints can prevent change for long periods in some directions. Punctuated equilibria may then arise by change in the developmental map, rather 
than only by change of the fitness landscape. In particular, gradual change in the developmental map may yield sudden path-peak creation events (Extended Data Fig. 2g-l; see also Fig. 3.4 of $\mathrm{Metz}^{42}$ ). Hence, punctuated equilibria may arise by gradual evolution of the developmental map, without revolutions in the developmental program.

Niche construction reshapes the path. Niche construction - whereby organisms affect their environment - has been suggested to be a developmental factor proposed to have major evolutionary effects ${ }^{12-14}$. How does niche construction affect evolution? We find that niche construction has a dual effect on adaptation. First, niche construction $\left(\partial \boldsymbol{\epsilon}^{\top} / \partial \mathbf{z}\right)$ reshapes the fitness landscape as previously recognised ${ }^{12-14}$. Second, niche construction reshapes the path on the landscape. To see this, we relax the assumption that niche construction is absent $\left(\partial \boldsymbol{\epsilon}^{\top} / \partial \mathbf{z} \neq \mathbf{0}\right)$, whilst maintaining our assumptions of no social development $\left(d \mathbf{x}^{\top} / d \overline{\mathbf{z}}=\mathbf{0}\right)$, and no exogenous environmental change $(\partial \boldsymbol{\epsilon} / \partial \tau=\mathbf{0})$. The evolutionary dynamics of the resident phenotype and gene expression are then given by

$$
\frac{\mathrm{d} \overline{\mathbf{z}}}{\mathrm{d} \tau}=\kappa \mathbf{G}_{\mathbf{z}} \frac{\delta w}{\delta \mathbf{z}},
$$

again provided that the developmental constraint (1) is met at all times. Eq. (9) depends on the semi-total selection gradient of the phenotype

$$
\frac{\delta w}{\delta \mathbf{z}}=\frac{\partial w}{\partial \mathbf{z}}+\frac{\partial \boldsymbol{\epsilon}^{\top}}{\partial \mathbf{z}} \frac{\partial w}{\partial \boldsymbol{\epsilon}},
$$

which measures semi-total selection considering environmental constraints but not developmental constraints. So, selection pushes uphill, no longer on the fitness landscape that ignores environmental constraints, but on the reshaped semi-total fitness landscape that considers such constraints (Fig. 2a-e). Eq. (10) shows that such reshaping of the fitness landscape 
is done by the interaction of niche construction and environmental sensitivity of selection ${ }^{43}$ $(\partial w / \partial \boldsymbol{\epsilon})$. The semi-total fitness landscape can have new peaks and valleys relative to the fitness landscape. Additionally, Eq. (9) depends on $\mathbf{G}_{\mathbf{z}}$, which is still singular but now depends on the interaction of niche construction and plasticity $\left(\partial \mathbf{x}^{\top} / \partial \boldsymbol{\epsilon}\right)$. Thus, development determines the path on the semi-total fitness landscape, but now this path is reshaped by the interaction of niche construction and plasticity (Fig. 2f-j). Consequently, niche construction reshapes the fitness landscape by interacting with environmental sensitivity of selection, and reshapes the admissible path on the landscape by interacting with phenotypic plasticity.

Social development alters the outcome. Extra-genetic inheritance, including social learning and epigenetics, has also been suggested to be a developmental factor proposed to have major evolutionary effects ${ }^{7,12,13}$. Extra-genetic inheritance can be seen as a particular form of social development $\left(d \mathbf{x}^{\top} / d \overline{\mathbf{z}} \neq \mathbf{0}\right)$, where the developed phenotype depends on the phenotype and gene expression of social partners. Social development can also mechanistically describe both indirect genetic effects, where the genes of social partners affect an individual's phenotype ${ }^{44}$, and phenotypic effects that need not stem from genes (e.g., if the individual's developed traits depend on social partners' environmentally induced developed traits). How does social development, including extra-genetic inheritance, affect evolution? We find that, since it alters the developmental map, social development alters the evolutionary outcome (i.e., the admissible evolutionary equilibria) by altering the admissible evolutionary path without necessarily altering the evolutionary equilibria themselves.

With social development, individuals with the same genotype experiencing the same en- 
vironment may develop different phenotypes even though development is assumed to be deterministic. This introduces a complication to evolutionary analysis, which we handle with the following notions. We say a gene expression, phenotype, and environment $\mathbf{m}^{* *}=\left(\mathbf{x}^{* *} ; \mathbf{y}^{* *} ; \boldsymbol{\epsilon}^{* *}\right)$ is a socio-devo equilibrium if and only if an individual with such gene expression and environment developing in the context of individuals having such gene expression, phenotype, and environment develops their same phenotype. A socio-devo equilibrium is socio-devo stable if and only if socio-devo stabilization dynamics ${ }^{17}$ are locally stable to small perturbations in the phenotype. Although social development can yield socio-devo unstable phenotypes, our analyses apply only to socio-devo stable phenotypes. Allowing for niche construction $\left(\partial \boldsymbol{\epsilon}^{\top} / \partial \mathbf{z} \neq \mathbf{0}\right)$ and social development $\left(\mathrm{d} \mathbf{x}^{\top} / \mathrm{d} \overline{\mathbf{z}} \neq \mathbf{0}\right.$ ), but assuming that there is no exogenous environmental change $(\partial \boldsymbol{\epsilon} / \partial \tau=\mathbf{0})$, then the evolutionary dynamics of the resident phenotype and gene expression are given by

$$
\frac{\mathrm{d} \overline{\mathbf{z}}}{\mathrm{d} \tau}=\kappa \mathbf{H}_{\mathbf{z}} \frac{\delta w}{\delta \mathbf{z}}=\kappa \mathbf{H}_{\mathbf{z y}} \frac{\mathrm{d} w}{\mathrm{~d} \mathbf{y}},
$$

again provided that the developmental constraint (1) is met at all times. The matrix $\mathbf{H}_{\mathbf{z}}$ is what we term the additive socio-genetic cross-covariance matrix of the geno-phenotype, which generalizes $\mathbf{G}_{\mathbf{z}}$ by including the effects of social development $\left(\mathbf{H}_{\mathbf{z}}\right.$ is similar to another generalization of the $\mathbf{G}$-matrix used in the indirect genetic effects literature ${ }^{44}$ ): while $\mathbf{G}_{\mathbf{z}}$ is the covariance matrix between breeding values, $\mathbf{H}_{\mathbf{z}}$ is the covariance matrix between socio-devo stabilized breeding values and breeding values. The matrix $\mathbf{H}_{\mathbf{z}}$ is always singular, so evolutionary stasis can again generally occur with persistent semi-total selection on the phenotype and gene expression under social development. In turn, the second equality in Eq. (11) depends on the matrix $\mathbf{H}_{\mathbf{z y}}$, which is the additive socio-genetic cross-covariance matrix between the geno- 
phenotype and gene expression, generalizing the corresponding matrix $\mathbf{G}_{\mathbf{z y}}$ we had in Eq. (8) to consider social development. Crucially and similarly to $\mathbf{G}_{\mathbf{z y}}$, this matrix $\mathbf{H}_{\mathbf{z y}}$ is non-singular if $\mathbf{G}_{\mathbf{y}}$ is non-singular. Thus, evolutionary equilibria still occur when total genetic selection vanishes $(\mathrm{d} w / \mathrm{d} \mathbf{y}=\mathbf{0})$ if there are no absolute mutational constraints. Social development may alter both the evolutionary equilibria (by altering $\mathrm{d} w / \mathrm{d} \mathbf{y}$ ) and the admissible path (by altering $\mathbf{H}_{\mathbf{z y}} ;$ Fig. 3). Alternatively, social development may not affect the evolutionary equilibria if total genetic selection $\mathrm{d} w / \mathrm{d} \mathbf{y}$ is independent of social partners' phenotype $\overline{\mathbf{z}}$, but social development may still affect the admissible evolutionary path given by the developmental constraint (1) (by altering only $\mathbf{H}_{\mathbf{z y}}$; Extended Data Fig. 3). In both cases, social development affects the admissible evolutionary equilibria and so the evolutionary outcome. When social development alters only the admissible path, it does so because of social developmental bias $\left(\partial \mathbf{x}^{\top} / \partial \overline{\mathbf{z}}\right.$; e.g., extra-genetic inheritance and indirect-genetic effects), and/or the interaction of social niche construction $\left(\partial \boldsymbol{\epsilon}^{\top} / \partial \overline{\mathbf{z}}\right)$ and plasticity $\left(\partial \mathbf{x}^{\top} / \partial \boldsymbol{\epsilon}\right)$ (from Layer 3, Eq. 3 of ref. $\left.{ }^{17}\right)$.

Plastic response alters the path. Research on the evolutionary effects of plasticity has increasingly intensified, with a particular focus on whether adaptive plastic change precedes genetic evolution $^{10}$. How does plasticity affect evolution? We find that plasticity also has a dual evolutionary effect. First, we have seen that plasticity $\left(\partial \mathbf{x}^{\top} / \partial \boldsymbol{\epsilon}\right)$ alters the evolutionary path by interacting with niche construction (i.e., endogenous environmental change; $\partial \boldsymbol{\epsilon}^{\top} / \partial \mathbf{z}$ ). This is because niche construction alters the environment, which through plasticity alters the developed phenotype and so the evolutionary path. Second, plasticity alters the evolutionary path by interacting with exogenous environmental change (e.g., eutrophication or climate change caused by other species; $\partial \boldsymbol{\epsilon} / \partial \tau)$. This is similarly because exogenous environmental change alters the 
environment, which through plasticity alters the developed phenotype and so the evolutionary path. With exogenous environmental change, the evolutionary dynamics comprise selection response and exogenous plastic response ${ }^{43}$, the latter of which modifies the developmental map and thus the evolutionary path as evolutionary time advances. To see this, assume now that there is niche construction $\left(\partial \boldsymbol{\epsilon}^{\top} / \partial \mathbf{z} \neq \mathbf{0}\right)$, social development $\left(\mathrm{d} \mathbf{x}^{\top} / \mathrm{d} \overline{\mathbf{z}} \neq \mathbf{0}\right)$, and exogenous environmental change $(\partial \boldsymbol{\epsilon} / \partial \tau \neq \mathbf{0})$. Then, the evolutionary dynamics of the resident phenotype and gene expression are given by

$$
\frac{\mathrm{d} \overline{\mathbf{z}}}{\mathrm{d} \tau}=\kappa \mathbf{H}_{\mathbf{z}} \frac{\delta w}{\delta \mathbf{z}}+\frac{\mathbf{s z}}{\mathbf{s} \boldsymbol{\epsilon}^{\top}} \frac{\partial \boldsymbol{\epsilon}}{\partial \tau}=\kappa \mathbf{H}_{\mathbf{z y}} \frac{\mathrm{d} w}{\mathrm{~d} \mathbf{y}}+\frac{\mathbf{s} \mathbf{z}}{\mathbf{s}^{\top}} \frac{\partial \boldsymbol{\epsilon}}{\partial \tau} .
$$

The matrix sz/s $\boldsymbol{\epsilon}^{\top}$ describes what we call stabilized plasticity of the phenotype and gene expression. Stabilized plasticity includes the effects of socio-devo stabilization and reduces to "total plasticity" $\mathrm{dz} / \mathrm{d} \boldsymbol{\epsilon}^{\top}$ if development is non-social. The first term in Eq. (12) is selection response $\left(\kappa \mathbf{H}_{\mathbf{z}} \delta w / \delta \mathbf{z}\right)$ whereas the second term is exogenous plastic response $\left(\left(\mathrm{sz} / \mathbf{s} \boldsymbol{\epsilon}^{\top}\right)(\partial \boldsymbol{\epsilon} / \partial \tau)\right)$, which makes the developmental map evolve as the environment exogenously changes (Fig. 4). Through exogenous plastic response, the evolutionary effects of development go beyond inducing genetic covariation. Moreover, Eq. (12) shows that exogenous plastic response can induce selection response, which allows for plastic change to preceed genetic change ${ }^{10}$. For example, if there is no selection response $\left(\mathbf{H}_{\mathbf{z}} \delta w / \delta \mathbf{z}=\mathbf{0}\right)$, exogenous plastic response causes change in the phenotype over evolutionary time, which generally changes $\mathbf{H}_{\mathbf{z}} \delta w / \delta \mathbf{z}$ at a subsequent evolutionary time thus inducing future selection response. In the idealized case where development is selective in the sense that the developmental map is a function of the selection gradient, stabilized plasticity can depend on the selection gradient. With selective development, exogenous plastic response can induce evolution either towards or away landscape peaks depending on 
the developmental constraints and initial conditions (Fig. 4). Additionally, exogenous plastic response with selective development has a limited ability of inducing evolution towards a landscape peak since plasticity must depend on developmental-past environments, which induces a developmental lag in the plastic response. Moreover, exogenous plastic response with selective development may induce evolution away a landscape peak when the rate of environmental change switches sign.

Development enables negative senescence. Thus far, we have considered evolutionary effects of development that occur even without considering that development takes time. Indeed, in our illustrative examples we have let age structure be collapsed so development happens instantaneously. We now show that explicit consideration of age progression in development may explain an otherwise puzzling ageing pattern.

Individuals may show senescence as they age, that is, decreasing survival or fertility after the onset of reproduction. Leading hypotheses for the evolution of senescence are based on the fact that the forces of selection decline with age ( $\phi_{a}$ and $\pi_{a}$ in Eq. 4$)$; thus, the reasoning has been that if a mutation has a beneficial effect early in life and a pleiotropic, deleterious effect of similar magnitude late in life, the mutation would be favoured ${ }^{19,45,46}$. The universality of declining forces of selection has suggested that senescence should be universal ${ }^{19}$, at least for organisms with a bodily reset at conception ${ }^{47}$. However, empirical research has increasingly reported organisms with negative senescence ${ }^{48}$, that is, where survival or fertility increase with age after the onset of reproduction, and models have suggested that this may stem from indeterminate growth ${ }^{47,49}$. 
We find that developmental propagation — whereby an early-acting mutation has a large phenotypic effect late in life - can drive the evolution of negative senescence. For example, if a mutation in gene expression has a deleterious effect at an early age $a$, so that $\delta w_{a} / \delta y_{i a}<0$, and a pleiotropic, beneficial effect of similar magnitude at a later age $j>a$, so that $\delta w_{j} / \delta x_{k j}>0$ for some phenotype, then semi-total selection is weaker at the later age because of declining selection forces $\left(\left|\delta w_{a} / \delta y_{i a}\right| \geq\left|\delta w_{j} / \delta x_{k j}\right|\right)$; yet, such late-beneficial mutation is favoured if total genetic selection on it is positive, that is, if

$$
\frac{\mathrm{d} w}{\mathrm{~d} y_{i a}}=\frac{\delta w_{a}}{\delta y_{i a}}+\frac{\mathrm{d} x_{k j}}{\mathrm{~d} y_{i a}} \frac{\delta w_{j}}{\delta x_{k j}}>0
$$

(Supplementary Information section 3), where the effect of the early mutation in gene expression on the late phenotype is

$$
\frac{\mathrm{d} x_{k j}}{\mathrm{~d} y_{i a}}=\frac{\delta \mathbf{x}_{a+1}^{\top}}{\delta y_{i a}}\left(\prod_{i=a+1}^{j-2} \frac{\delta \mathbf{x}_{i+1}^{\top}}{\delta \mathbf{x}_{i}}\right) \frac{\delta x_{k j}}{\delta \mathbf{x}_{j-1}}
$$

(from equation E17 of ref. ${ }^{17}$ ). This effect of the early mutation in gene expression on the late phenotype may increase with the phenotype age $j$ because of developmental propagation of phenotypic effects as age advances, a propagation that is described by the term in parentheses in Eq. (14). So total genetic selection may increase with the age $j$ at which the pleiotropic effect is expressed. Consequently, a mutation with late beneficial effects may be favoured despite early deleterious effects. Thus, developmental propagation may enable selection to shape traits at advanced age, enabling adaptive evolution of increasing survival or fertility as ageing proceeds despite decreasing selection forces after the onset of reproduction. 


\section{Discussion}

We have shown that development affects evolution by determining the evolutionary path. This sharpens the basic principle of adaptation as the climbing of the fitness landscape ${ }^{1}$ (sensu Dieckmann and $\mathrm{Law}^{50}$ ), such that evolutionary outcomes occur at peaks on the path rather than on the landscape. Developmental constraints thus stop evolution at path peaks that can happen mid-way on the fitness landscape. Selective development, if feasible, can bring some of the path closer to the landscape peak by means of exogenous plastic response. Our findings substantiate several although not all intuitions previously given in relation to the role of development in evolution ${ }^{8-13}$. In particular, seemingly disparate development-related factors previously suggested to be evolutionarily important ${ }^{8-13}$ variously alter the evolutionary path and hence also the outcome. Our analysis yields answers to various major questions, namely the origin of diversity, the punctuated fossil record, the paradox of stasis, the rarity of stabilizing selection, and the origin of negative senescence. Our findings entail that an understanding of development is instrumental for evolutionary understanding: change in development alone changes evolutionary equilibria and the admissible path by changing absolute socio-genetic constraints $\left(\mathbf{H}_{\mathbf{z}}\right)$, thereby changing evolutionary outcomes even if directional selection $(\partial w / \partial \mathbf{z})$, total selection ( $\mathrm{d} w / \mathrm{d} \mathbf{z}$ or $\mathrm{d} w / \mathrm{d} \mathbf{y}$ ), or the non-social environment $(\boldsymbol{\epsilon})$ remain constant (Extended Data Fig. 3). Overall, our results show that development has major evolutionary consequences.

1. Lande, R. Quantitative genetic analysis of multivariate evolution applied to brain: body size allometry. Evolution 34, 402-416 (1979).

2. Charlesworth, B., Lande, R. \& Slatkin, M. A neo-Darwinian commentary on macroevolu- 
tion. Evolution 36, 474-498 (1982).

3. Cheverud, J. M. Quantitative genetics and developmental constraints on evolution by selection. J. Theor. Biol. 110, 155-171 (1984).

4. Maynard Smith, J. et al. Developmental constraints and evolution. Q. Rev. Biol. (1985).

5. Klingenberg, C. P. Evolution and development of shape: integrating quantitative approaches. Nat. Rev. Genet. 11, 623-635 (2010).

6. Fisher, R. XV.-The correlation between relatives on the supposition of Mendelian inheritance. Trans. Roy. Soc. Edinb. 52, 399-433 (1918).

7. Baldwin, J. M. A new factor in evolution. Am. Nat. 30, 441-451 (1896).

8. Waddington, C. H. Evolutionary adaptation. Perspect. Biol. Med. 2, 379-401 (1959).

9. Gould, S. J. \& Lewontin, R. C. The spandrels of San Marco and the Panglossian paradigm: a critique of the adaptationist programme. Proc. R. Soc. Lond. B 205, 581-598 (1979).

10. West-Eberhard, M. J. Developmental Plasticity and Evolution (Oxford Univ. Press, Oxford, UK, 2003).

11. Pigliucci, M. Do we need an extended evolutionary synthesis? Evolution 61, 2743-2749 (2007).

12. Laland, K. et al. Does evolutionary theory need a rethink? Yes, urgently. Nature 514, 161-164 (2014). 
13. Laland, K. N. et al. The extended evolutionary synthesis: its structure, assumptions and predictions. Proc. R. Soc. B 282, 20151019 (2015).

14. Odling-Smee, F. J., Laland, K. N. \& Feldman, M. W. Niche construction. Am. Nat. 147, 641648 (1996).

15. Arthur, W. Biased Embryos and Evolution (Cambridge Univ. Press, Cambridge, UK, 2004).

16. Pigliucci, M. \& Schlichting, C. D. On the limits of quantitative genetics for the study of phenotypic evolution. Acta Biotheor. 45, 143-160 (1997).

17. González-Forero, M. \& Gardner, A. A mathematical framework for evo-devo dynamics. Preprint: https://www.biorxiv.org/content/10.1101/2021.05.17.444499v2.

18. Lande, R. A quantitative genetic theory of life history evolution. Ecology 63, 607-615 (1982).

19. Hamilton, W. D. The moulding of senescence by natural selection. J. Theor. Biol. 12, 12-45 (1966).

20. Lande, R. The genetic covariance between characters maintained by pleiotropic mutations. Genetics 94, 203-215 (1980).

21. Wagner, G. P. On the eigenvalue distribution of genetic and phenotypic dispersion matrices: Evidence for a nonrandom organization of quantitative character variation. J. Math. Biol. 21, 77-95 (1984).

22. Barton, N. H. \& Turelli, M. Adaptive landscapes, genetic distance and the evolution of quantitative characters. Genet. Res. 49, 157-173 (1987). 
23. Lynch, M. \& Walsh, B. Genetics and Analysis of Quantitative Traits (Sinauer, Sunderland, MA, USA, 1998).

24. Houle, D. Genetic covariance of fitness correlates: what genetic correlations are made of and why it matters. Evolution 45, 630-648 (1991).

25. Kirkpatrick, M. \& Lofsvold, D. Measuring selection and constraint in the evolution of growth. Evolution 46, 954-971 (1992).

26. Altenberg, L. Genome growth and the evolution of the genotype-phenotype map. In Banzhaf, W. \& Eeckman, F. H. (eds.) Evolution and biocomputation, vol. 899 of Lecture Notes in Computer Science, 205-259 (Springer-Verlag, 1995).

27. Merilä, J., Sheldon, B. \& Kruuk, L. Explaining stasis: microevolutionary studies in natural populations. Genetica 112, 199-222 (2001).

28. Kirkpatrick, M. Patterns of quantitative genetic variation in multiple dimensions. Genetica 136, 271-284 (2009).

29. Kingsolver, J. G. \& Diamond, S. E. Phenotypic selection in natural populations: What limits directional selection? Am. Nat. 177, 346-357 (2011).

30. Morrissey, M. B. Selection and evolution of causally covarying traits. Evolution 68, 17481761 (2014).

31. Morrissey, M. B. Evolutionary quantitative genetics of nonlinear developmental systems. Evolution 69, 2050-2066 (2015). 
32. Barton, N. H., Etheridge, A. M. \& Véber, A. The infinitesimal model: definition, derivation, and implications. Theor. Popul. Biol. 118, 50-73 (2017).

33. Kingsolver, J. G. et al. The strength of phenotypic selection in natural populations. Am. Nat. 157, 245-261 (2001).

34. Walsh, B. \& Lynch, M. Evolution and Selection of Quantitative Traits (Oxford Univ. Press, Oxford, UK, 2018).

35. Gavrilets, S. \& Losos, J. B. Adaptive radiation: Contrasting theory with data. Science 323, $732-737$ (2009).

36. Geritz, S. A. H., Kisdi, E., Meszéna, G. \& Metz, J. A. J. Evolutionarily singular strategies and the adaptive growth and branching of the evolutionary tree. Evol. Ecol. 12, 35-57 (1998).

37. Eldredge, N. \& Gould, S. J. Punctuated equilibria: an alternative to phyletic gradualism. In Thomas, J. M. (ed.) Models in Paleobiology, 82-115 (Freeman, Cooper and Company, 1972).

38. Hunt, G., Hopkins, M. J. \& Lidgard, S. Simple versus complex models of trait evolution and stasis as a response to environmental change. Proc. Natl. Acad. Sci. USA 112, 4885-4890 (2015).

39. Gould, S. J. Is a new and general theory of evolution emerging? Paleobiology 6, 119-130 (1980).

40. Beldade, P., Koops, K. \& Brakefield, P. M. Developmental constraints versus flexibility in morphological evolution. Nature 416, 844-847 (2002).

41. Eldredge, N. et al. The dynamics of evolutionary stasis. Paleobiology 31, 133-145 (2005). 
42. Metz, J. A. J. Thoughts on the geometry of meso-evolution: collecting mathematical elements for a postmodern synthesis. In Chalub, F. A. C. C. \& Rodrigues, J. F. (eds.) The Mathematics of Darwin's Legacy, 193-231 (Springer, 2011).

43. Chevin, L.-M., Lande, R. \& Mace, G. M. Adaptation, plasticity, and extinction in a changing environment: Towards a predictive theory. PLOS Biology 8, 1-8 (2010).

44. Moore, A. J., Brodie III, E. D. \& Wolf, J. B. Interacting phenotypes and the evolutionary process: I. direct and indirect genetic effects of social interactions. Evolution 51, 1352-1362 (1997).

45. Medawar, P. B. An unsolved problem of biology (H. K. Lewis, London, UK, 1952).

46. Williams, G. C. Natural selection, and the evolution of senescence. Evolution 11, 398-411 (1957).

47. Lehtonen, J. Longevity and the drift barrier: bridging the gap between Medawar and Hamilton. Evol. Lett. 4, 382-393 (2020).

48. Jones, O. R. et al. Diversity of ageing across the tree of life. Nature 505, 169-173 (2014).

49. Vaupel, J. W., Baudisch, A., Dölling, M., Roach, D. A. \& Gampe, J. The case for negative senescence. Theor. Pop. Biol. 65, 339-351 (2004).

50. Dieckmann, U. \& Law, R. The dynamical theory of coevolution: a derivation from stochastic ecological processes. J. Math. Biol. 34, 579-612 (1996).

51. Schluter, D. Adaptive radiation along genetic lines of least resistance. Evolution 50, 17661774 (1996). 
Supplementary Information is available in the online version of the paper.

Acknowledgements We thank K.N. Laland, R. Lande, L.C. Mikula, A.J. Moore, and M.B. Morrissey for comments on previous versions of the manuscript, and D.M. Shuker for feedback. We apologise for omitting citations due to space constraints. This work was funded by an ERC Consolidator Grant to AG (grant no. 771387). AG was also funded by a NERC Independent Research Fellowship (grant no. NE/K009524/1).

Author Contributions M.G.F. conceived the project, designed the mathematical framework, and derived the results. M.G.F. and A.G. interpreted the results and wrote the paper.

Author Information The authors declare no competing financial interests. Correspondence and requests for materials should be addressed to MGF (mgf3@st-andrews.ac.uk). 
bioRxiv preprint doi: https://doi.org/10.1101/2021.10.20.464947; this version posted October 21, 2021. The copyright holder for this preprint (which was not certified by peer review) is the author/funder, who has granted bioRxiv a license to display the preprint in perpetuity. It is made available under aCC-BY-NC 4.0 International license.

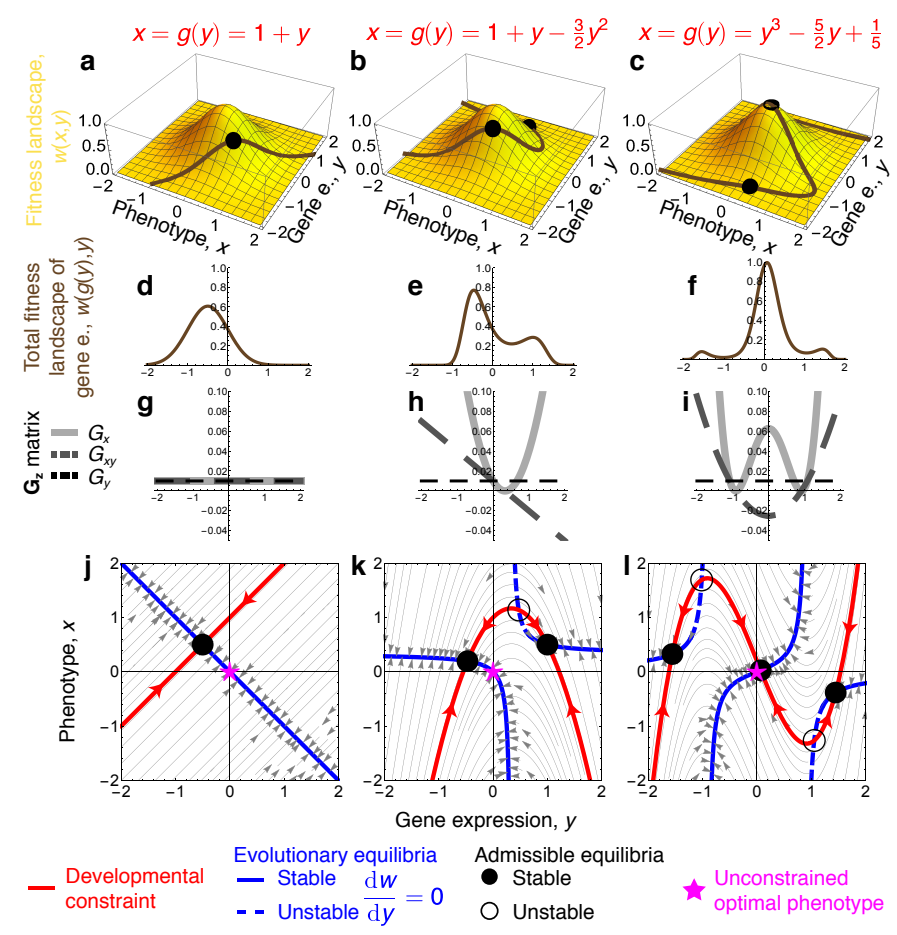

Figure 1: Caption is on the next page. 
Figure 1: Development determines the evolutionary path. a-c, Fitness landscape for a phenotype $x$ and a gene product's expression level $y$, with three developmental maps. The developmental map determines the path on the landscape as $x$ can only take values along the path. d-f, The total fitness landscape of gene expression gives the elevation profile considering developmental and environmental constraints. g-i, Additive genetic variances and covariances depend on the developmental map and evolve as gene expression evolves. $\mathbf{j}-\mathbf{l}$, The evolutionary dynamics follow the trajectory given by the developmental map (red; gray arrows are parallel or antiparallel to leading eigenvectors of $\mathbf{G}_{\mathbf{z}}$, called "genetic lines of least resistance" ${ }^{51}$ ). Evolutionary outcomes (black dots) depend on the developmental map, and are at path peaks rather than landscape peaks. The number of evolutionary equilibria (blue) is infinite because $\mathbf{G}_{\mathbf{z}}$ is singular; evolutionary equilibria occur when total genetic selection vanishes where the selection gradient is generally non-zero. The intersection of the developmental map and evolutionary equilibria yields the admissible evolutionary equilibria. Different developmental maps yield different evolutionary trajectories and outcomes with the same single-peak landscape. Developmental bias is quantified by the slope of the red line $(\partial x / \partial y)$. Evolutionary change satisfies $\mathrm{d} \overline{\mathbf{z}} / \mathrm{d} \tau=\mathbf{G}_{\mathbf{z}} \partial w / \partial \mathbf{z}$, where $\mathbf{G}_{\mathbf{z}}=(\mathrm{d} \mathbf{z} / \mathrm{d} y) G_{y}\left(\mathrm{~d} \mathbf{z}^{\top} / \mathrm{d} y\right)=(\mathrm{d} x / \mathrm{d} y, 1)^{\top} G_{y}(\mathrm{~d} x / \mathrm{d} y, 1)=$ $G_{y}\left((\mathrm{~d} x / \mathrm{d} y)^{2}, \mathrm{~d} x / \mathrm{d} y ; \mathrm{d} x / \mathrm{d} y, 1\right)=\left(G_{x}, G_{x y} ; G_{x y}, G_{y}\right)$, which is singular. The additive genetic variance of $x$ is $G_{y}(\mathrm{~d} x / \mathrm{d} y)^{2}$. The total effects of a mutant's gene expression on her geno-phenotype are $\mathrm{d} \mathbf{z}^{\top} / \mathrm{d} y=(\mathrm{d} x / \mathrm{d} y, \mathrm{~d} y / \mathrm{d} y)=(\mathrm{d} x / \mathrm{d} y, 1)$. Fitness is $w(x, y)=\exp \left(-x^{2}-y^{2}\right)$ and mutational variance is $G_{y}=0.01$. Distinguishing mutant from resident is here unnecessary because of vanishing mutational variation and no social interactions. Functions used are for illustration purposes and do not describe specific systems. 


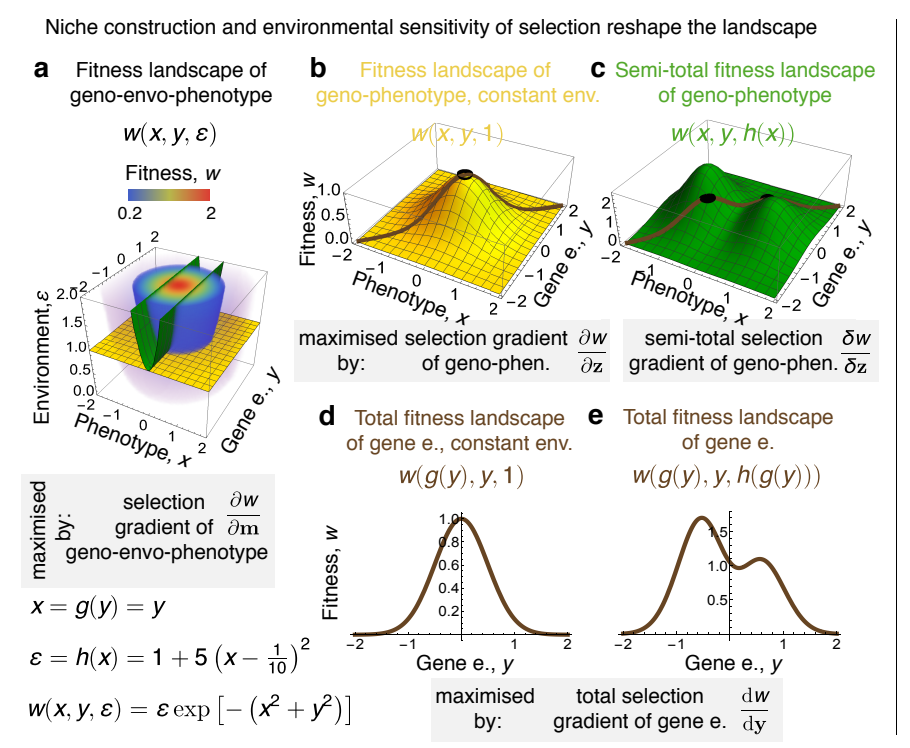

Figure 2: Niche construction reshapes the fitness landscape and the evolutionary path. a-e,

There is niche construction by the phenotype $x$ which non-linearly affects the environmental variable $\epsilon$, which in turn increases fitness. a, Fitness landscape vs. phenotype, gene expression, and environment. Slices for constant environment (yellow) and for the environmental constraint (green) are respectively shown in $\mathbf{b}$ and $\mathbf{c}$. The interaction of niche construction $(\partial \epsilon / \partial x)$ and environmental sensitivity of selection $(\partial w / \partial \epsilon)$ reshapes the landscape (from $\mathbf{b}$ to $\mathbf{c}$ ) by affecting semi-total selection $\delta w / \delta \mathbf{z}$ but it does not affect genetic covariation $\mathbf{G}_{\mathbf{z}} \cdot \mathbf{f} \mathbf{- j}$, There is niche construction by gene expression $y$ which linearly affects the environmental variable $\epsilon$, which in turn increases the phenotype. The interaction of niche construction $(\partial \epsilon / \partial y)$ and plasticity $(\partial x / \partial \epsilon)$ reshapes the path (from $\mathbf{g}$ to $\mathbf{h})$. This interaction affects genetic covariation $\mathbf{G}_{\mathbf{z}}$ but does not affect semi-total selection $\delta w / \delta \mathbf{z}$. In all panels, evolutionary change satisfies $\mathrm{d} \overline{\mathbf{z}} / \mathrm{d} \tau=\mathbf{G}_{\mathbf{z}} \delta w / \delta \mathbf{z}$, where $\delta w / \delta \mathbf{z}=\partial w / \partial \mathbf{z}+(\partial \epsilon / \partial \mathbf{z})(\partial w / \partial \epsilon)$ and $\mathbf{G}_{\mathbf{z}}$ is given in Fig. 1. In a-e, $\mathrm{d} x / \mathrm{d} y=\partial x / \partial y$, whereas in $\mathbf{f}-\mathbf{j}, \mathrm{d} x / \mathrm{d} y=\partial x / \partial y+(\partial \epsilon / \partial y)(\partial x / \partial \epsilon)$. 


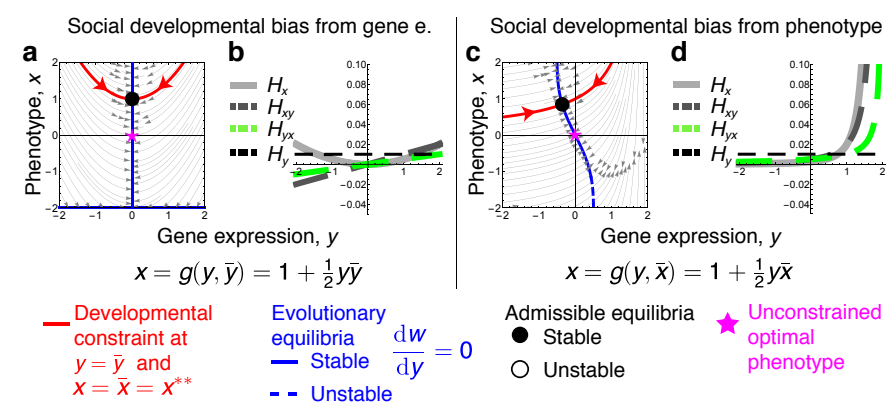

Figure 3: Social development alters the evolutionary outcome. a-b, There is social development, particularly social developmental bias from gene expression $(\partial x / \partial \bar{y} \neq 0)$. Social development introduces a non-linearity relative to the developmental map of Fig. 1a,d,g,j, which changes the evolutionary equilibria (blue), admissible path (red), and outcome (solid dot). cd, As in $\mathbf{a}-\mathbf{b}$, but there is social developmental bias from the phenotype $(\partial x / \partial \bar{x} \neq 0)$. The developmental map is that of $\mathbf{a}-\mathbf{b}$ replacing $\bar{y}$ with $\bar{x}$. This changes the evolutionary equilibria, admissible path, and outcome. In all panels, evolutionary change satisfies $\mathrm{d} \overline{\mathbf{z}} / \mathrm{d} \tau=\mathbf{H}_{\mathbf{z}} \partial w / \partial \mathbf{z}$, where $w(x, y)=\exp \left(-x^{2}-y^{2}\right)$ and $\mathbf{H}_{\mathbf{z}}=(\mathrm{sz} / \mathrm{s} y) G_{y}\left(\mathrm{~d} \mathbf{z}^{\top} / \mathrm{d} y\right)=\left(H_{x x}, H_{x y} ; H_{y x}, H_{y y}\right)$, with sz/s $y=$ $(\mathrm{s} x / \mathrm{s} y ; 1)$. For a-b, $\mathrm{s} x / \mathrm{s} y=\mathrm{d} x / \mathrm{d} y+\mathrm{d} x / \mathrm{d} \bar{y}$. For c-d, $\mathrm{s} x / \mathrm{s} y=(1-\partial x / \partial \bar{x})^{-1} \mathrm{~d} x / \mathrm{d} y$, and $\mathrm{d} \mathbf{z}^{\top} / \mathrm{d} y$ and $s x / s y$ are evaluated at the socio-devo equilibrium $x^{* *}$ that solves $x^{* *}=g\left(y, x^{* *}\right)$. Since development is social, a fitness landscape visualization as in Fig. 1a is not possible. In c, the stream plot (gray arrows) is only locally accurate around the developmental constraint where the socio-devo equilibrium holds. 


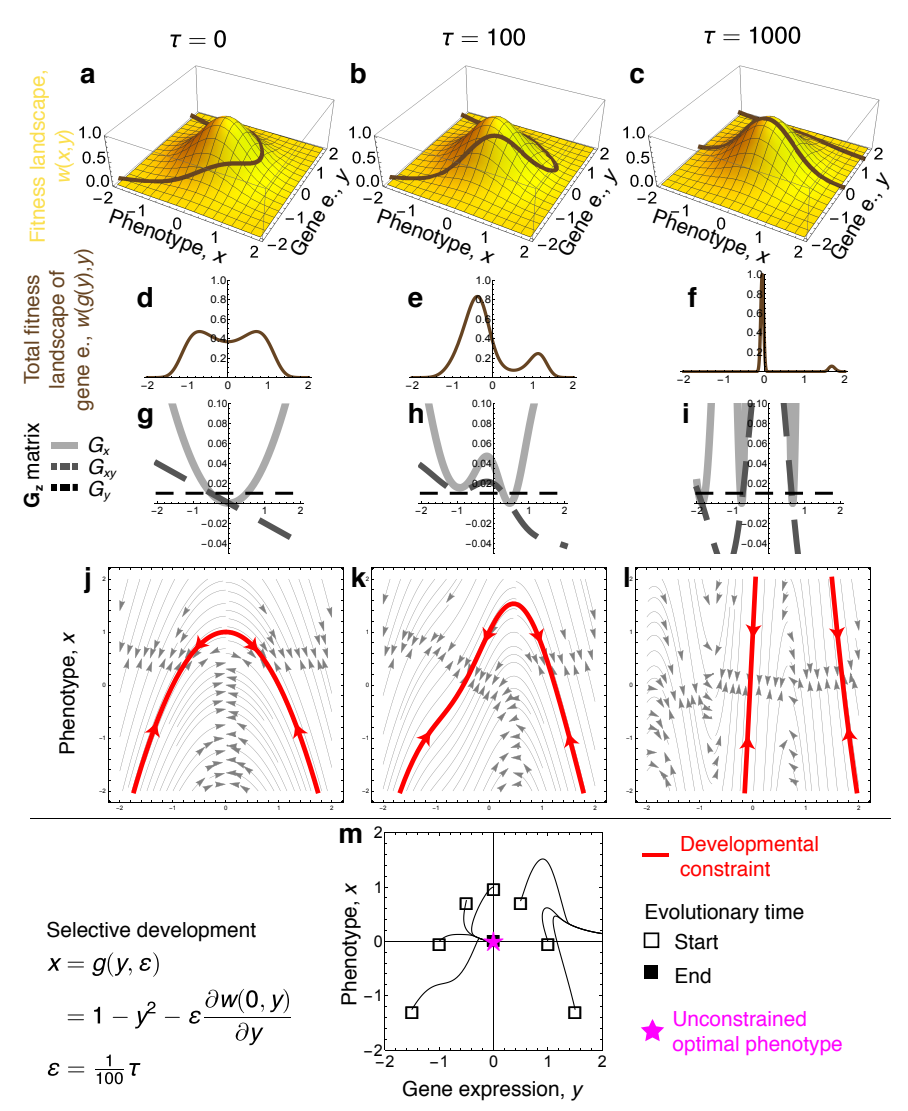

Figure 4: Exogenous plastic response changes the evolutionary path. We let there be exogenous environmental change and plasticity, but no niche construction, so plasticity does not affect $\mathbf{G}_{\mathbf{z}}$ but only the exogenous plastic response. Development is selective by letting plasticity equal the selection gradient at the optimal phenotype, $\partial x / \partial \epsilon=\partial w(0, y) / \partial y$. a-l, Exogenous environmental change induces exogenous plastic response which raises part of the path on the landscape. m, Resulting evolutionary dynamics showing that selective development induces some evolutionary trajectories to converge to the landscape peak, whereas others do not depending on their initial condition due to the quadratic developmental constraint. Evolutionary change satisfies $\mathrm{d} \overline{\mathbf{z}} / \mathrm{d} \tau=\mathbf{G}_{\mathbf{z}} \partial w / \partial \mathbf{z}+(\mathrm{d} \mathbf{z} / \mathrm{d} \epsilon)(\partial \epsilon / \partial \tau)$, where $\mathrm{d} \mathbf{z} / \mathrm{d} \epsilon=(\mathrm{d} x / \mathrm{d} \epsilon ; \mathrm{d} y / \mathrm{d} \epsilon)=(\partial x / \partial \epsilon ; 0)$ and $\mathbf{G}_{\mathbf{z}}$ is given in Fig. 1. Fitness is $w(x, y)=\exp \left(-x^{2}-y^{2}\right)$. 


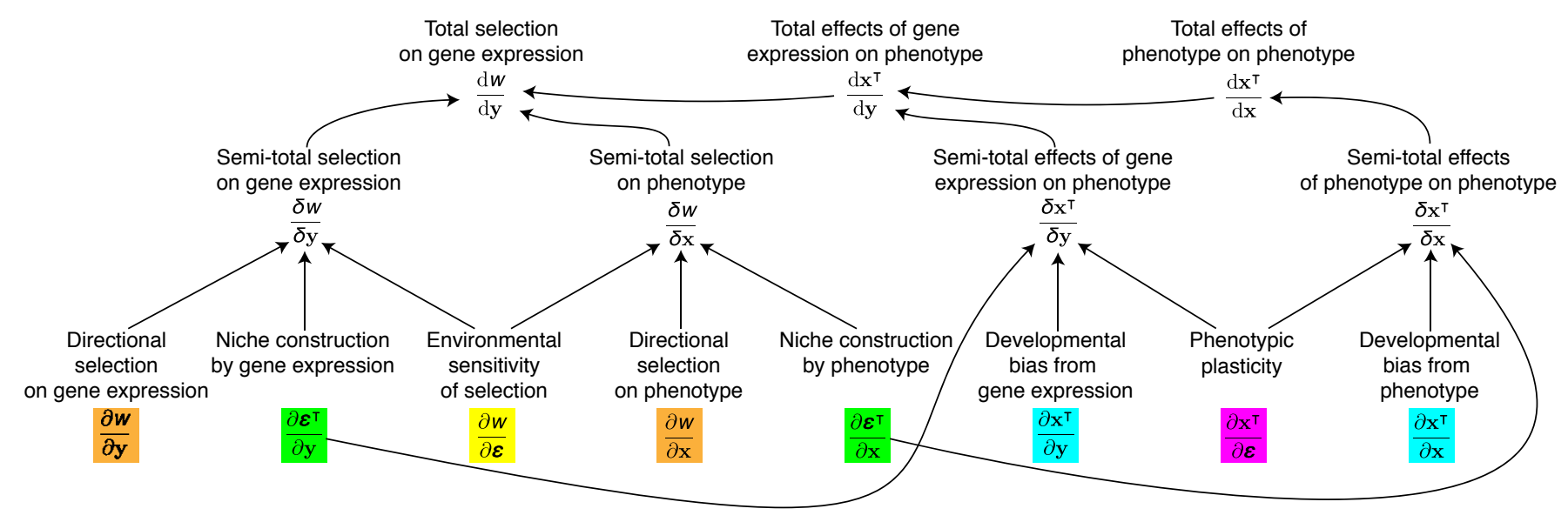

Extended Data Figure 1: Total genetic selection depends on many factors. Total genetic selection - which is measured by the total selection gradient of gene expression $\mathrm{d} w / \mathrm{d} \mathbf{y}-\mathrm{describes}$ selection response relatively completely in that it is premultiplied by a non-singular matrix $\mathbf{H}_{\mathbf{z y}}$ if there are no absolute mutational constraints, so total genetic selection can identify evolutionary equilibria. In contrast, direct phenotypic and genetic selection - measured by $\partial w / \partial \mathbf{z}$ — and total phenotypic and genetic selection - measured by $\mathrm{d} w / \mathrm{d} \mathbf{z}-$ describe selection response less completely in that they are always premultiplied by a singular matrix and so do not generally identify evolutionary equilibria. Total genetic selection depends on direct directional selection, developmental bias, plasticity, niche construction, and environmental sensitivity of selection. An arrow from a variable to another one indicates that the latter depends on the former. 


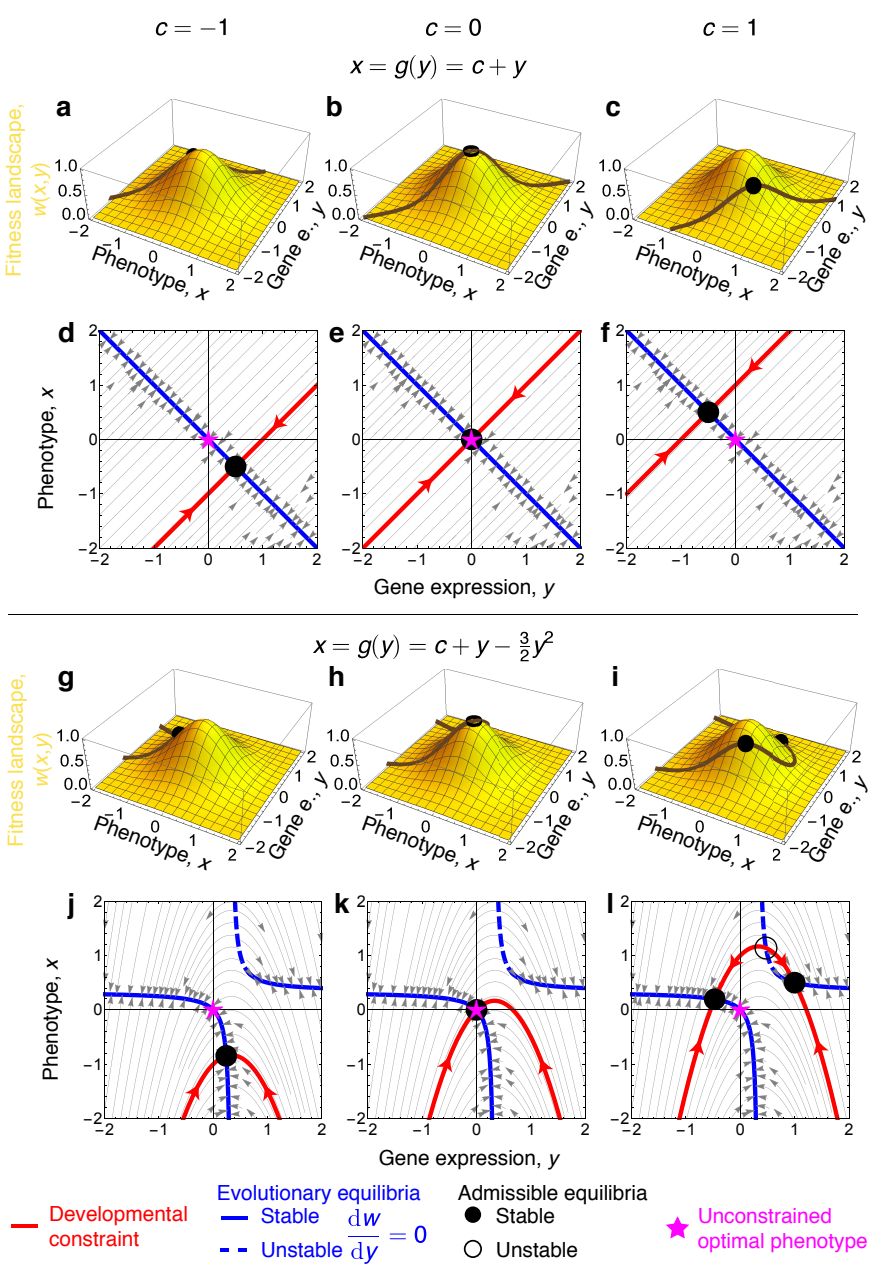

Extended Data Figure 2: Development-driven diversification. Development may drive pheno-

typic diversification in two ways. First, by shifting path peaks (a-f). Second, by creating path peaks (g-l). The evolutionary dynamics are given as in the legend of Fig. 1. As $c$ changes, development $g$ changes, but total developmental bias $\mathrm{d} x / \mathrm{d} y$ does not change, so the evolutionary equilibria remain the same (blue); however, the admissible evolutionary equilibria do change (open and solid circles) and so the outcome even though the fitness landscape is constant and single peaked. 

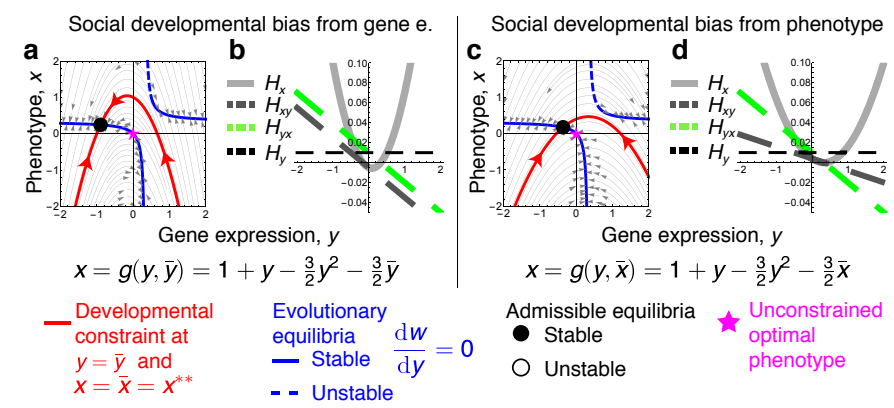

Extended Data Figure 3: Social development can alter the evolutionary outcome without al-

tering evolutionary equilibria. The plots are as in Fig. 3 with the developmental maps being a linear modification of those in Fig. 1b,e,h,k. Relative to that figure, social development does not alter total developmental bias $\mathrm{d} x / \mathrm{d} y$ so it does not affect evolutionary equilibria (blue). However, social development affects the admissible path (red) and so the evolutionary outcome (solid dot). Note $H_{x}$ can be negative in $\mathbf{b}$ as it gives the covariance between the stabilized breeding value of $x$ and the breeding value of $x$ (in contrast, $G_{x}$ gives the variance of the breeding value of $x$, which is non-negative). 